\title{
Aplicación de la energía solar térmica para la reducción del consumo eléctrico en un sistema de calentamiento de agua sanitaria industrial
}

Alex Ramires ${ }^{1}$, Jorge Cavalchini ${ }^{2}$, Andres Carballo ${ }^{3}$, Norberto Odobez ${ }^{4}$ ( $)$

RESUMEN: El creciente aumento de los costos de la energía por medio de combustibles fósiles, el impulso en el país de la Energías Renovables y la Generación Distribuida hacen considerar a las empresas la sustitución de las primeras por las alternativas tecnológicas disponibles de energías renovables. E1 objetivo de este trabajo fue reducir los costos y el impacto ambiental en el calentamiento del agua sanitaria del edificio administrativo, vestuarios y comedor de BAYER ZARATE, realizado con energía eléctrica, por medio de colectores solares térmicos de tecnología de tubo de vacío. Este proyecto se enmarcó en una Práctica Profesional Supervisada - UTN en industria, obligatoria en el último año de la carrera. La tutoría fue realizada por el Centro CEA y el Departamento de Ingeniería Mecánica, resulta de sumo interés este formato ya que hace interactuar la investigación y docencia con la industria en forma directa. Para la primera etapa del proyecto se realizó el diseño de forma convencional y una comparativa utilizando TRNSYS18; concluyendo, se instalaron seis unidades de 30 tubos cada una, superficie de apertura de $17 \mathrm{~m} 2$. Resultando un ahorro anual en energía eléctrica de $16374 \mathrm{kWh}$, que corresponde al 30\% del total, amortización 10 años y dejar de emitir un total de 8,762 $\mathrm{tn} / \mathrm{CO} 2$ por año.

Palabras claves: Energía solar térmica, ahorro de energía, simulación, concientizar.

(*) e-mail: odobezn@frd.utn.edu.ar

1 Alumno Departamento Ingeniería Mecánica, Facultad Regional Delta - UTN, ramires.alexis1@gmail.com 2 Director Departamento Ingeniería Mecánica, Facultad Regional Delta - UTN, jcavalchini@frd.utn.edu.ar 3 Investigador Centro CEA, Facultad Regional Delta-UTN, acarballo@frd.utn.edu.ar 4 Director Centro CEA, Facultad Regional Delta-UTN, odobezn@frd.utn.edu.ar 


\section{ABSTRACT:}

he growing increase of the costs of the energy by means of fossil fuels, the impulse in the country of the Renewable Energies and the Distributed Generation make consider to the companies the substitution of the first for the available technological alternatives of renewable energies. The objective of this work was to reduce the costs and the environmental impact in the heating of the sanitary water of the administrative building, changing rooms, bathrooms and canteen of BAYER ZARATE, made with electrical energy, by means of thermal solar collectors of vacuum tube technology. This project was framed in a Supervised Professional Practice - UTN in industry, mandatory in the last year of the race. The tuto- ring was carried out by the CEA Center and the Department of Mechanical Engineering. This format is of great interest as it makes direct interaction between research and teaching with the industry. For the first stage of the project, the design was carried out in a conventional manner and a comparative one using TRNSYS18; concluding, six units of 30 tubes each were installed, opening area of $17 \mathrm{~m} 2$. Resulting in an annual saving in electricity of $16374 \mathrm{kWh}$, which corresponds to $30 \%$ of the total, amortization 10 years and stop issuing a total of 8,762tn/CO2 per year.

Keywords: Solar thermal energy, energy saving, simulation, make aware

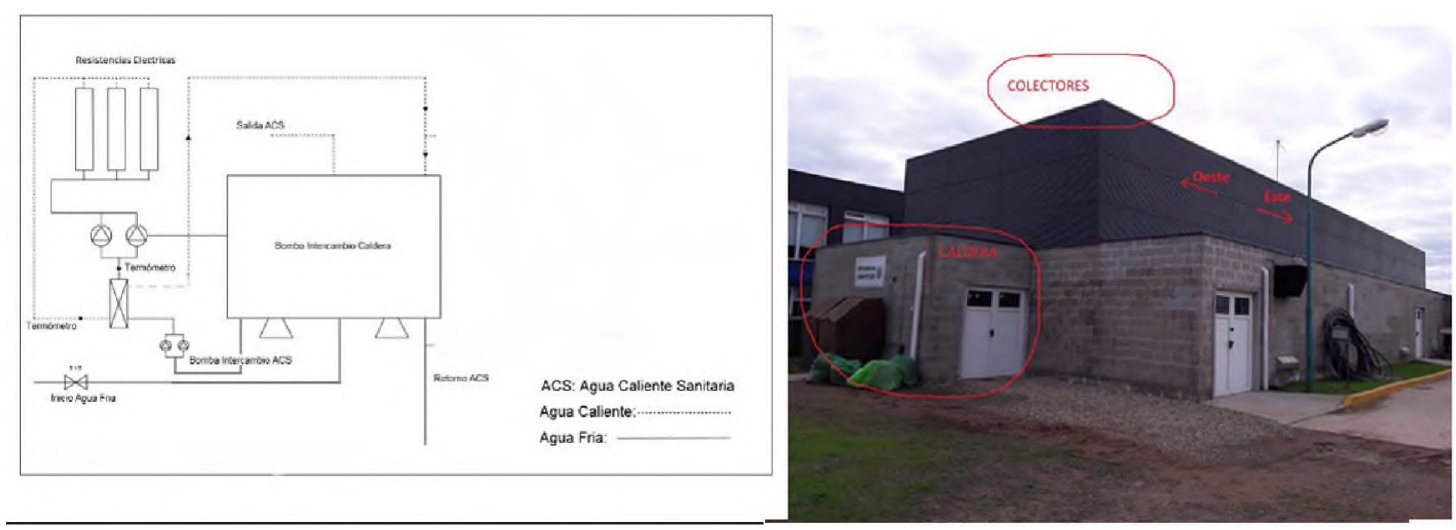

Figura 1: a) Plano de la instalación del circuito de resistencias. b) Edificio caldera

\section{INTRODUCCIÓN}

El objetivo de este trabajo fue reducir los costos y hacer un aporte al medioambiente, en el calentamiento del agua sanitaria del edificio administrativo, vestuarios y comedor de BAYER ZARATE, cubriendo parte de esta demanda de calor por medio del aporte de agua caliente con colectores solares térmicos.

\section{INSTALACIÓN ACTUAL}

La instalación está formada por un tanque, aislado, de 4500 lts. de agua la cual se calienta mediante un circuito cerrado de 3 
resistencias eléctricas, con una potencia total instalada de 36.000 Watts. El tanque se encuentra en un recinto ubicado en el edificio administrativo de Bayer Cropscience Planta Zarate; Latitud: $-34,07$, Longitud $-59,05$. Ver Figura 1 a) y b).

\section{Condiciones iniciales de cálculo}

A1 no contar con instrumentos que permitan determinar cuánto tiempo están funcionando las resistencias eléctricas ni cuánto es su consumo y tampoco con un medidor de caudal para determinar la cantidad de agua consumida. Se debió realizar un análisis para el cálculo de los colectores considerando como punto de partida las estimaciones de consumo de agua y por ende el consumo eléctrico para calentar dicha agua, teniendo en cuenta los horarios de uso específico de la misma.

Se parte entonces de considerar: El uso principal es para la ducha de aproximadamente 80 personas por día, en 3 turnos (mañana, tarde y noche). El valor asumido de 50 litros por persona, que excede el mencionado en (1) de 30 litros por persona para vestuarios/duchas, es debido a que se estimó un consumo adicional de agua caliente en los baños y en el comedor de planta, que también prevé el sistema. Con un valor de temperatura de almacenamiento de $45^{\circ} \mathrm{C}$ de diciembre a marzo y de $60^{\circ} \mathrm{C}$ para el resto del año. En tabla 1 se preestablecen los parámetros de radiación local (2), temperatura del agua de provisión en la localización del proyecto (3), utilizados para el cálculo.

\begin{tabular}{|l|l|l|l|l|l|l|l|l|l|l|l|l|}
\hline Mes & 1 & 2 & 3 & 4 & 5 & 6 & 7 & 8 & 9 & 10 & 11 & 12 \\
\hline $\begin{array}{l}\text { Temperatura } \\
\text { agua de } \\
\text { provisión }{ }^{\circ} \mathrm{C}\end{array}$ & 22 & 22 & 18 & 16 & 16 & 12 & 12 & 12 & 16 & 16 & 18 & 22 \\
\hline $\begin{array}{l}\text { Radiación solar } \\
\text { horizontal } \\
\mathrm{kWh} \text { /m2dia. }\end{array}$ & 6,50 & 5,50 & 4,50 & 3,00 & 2,50 & 2,00 & 2,00 & 3,00 & 4,00 & 5,00 & 6,00 & 6,50 \\
\hline
\end{tabular}

Tabla I - Datos de temperatura ambiente, temperatura del agua y radiación promedio mensual

Los datos climatológicos y de radiación solar para la región se corroboraron con los arrojados por la estación meteorológica que tiene instalada la Facultad Regional Delta en su centro CEA: http:// meteo.frd.utn.edu.ar/ distancia del proyecto $30 \mathrm{Km}$.

\section{CÁLCULO DE LA DEMANDA ENERGÉTICA}

Para calcular la Energía requerida en cada época del año para llegar a la tempe- ratura deseada y en función de su uso, se utilizarán los siguientes datos: Cantidad de Personas 80, Consumo de Agua Caliente Sanitaria por Persona 50L/día. Necesidad de agua caliente total 4000L/día, dividida en dos períodos uno nocturno 2500L/día y otro diurno de $1500 \mathrm{~L} /$ día. Se realizó el cálculo de la energía necesaria mes a mes aplicando $\mathrm{Q}=\mathrm{m} \times \mathrm{Ce}$

$\left(\mathrm{t}^{\circ} \mathrm{f}-\mathrm{t}^{\circ} \mathrm{i}\right)$, con $\mathrm{Q}[\mathrm{Kcal}], \mathrm{m}=1 \mathrm{~kg}$ agua $\approx$ 1lts agua y $\mathrm{Ce}=1 \mathrm{kcal} / \mathrm{kg}^{\circ} \mathrm{C}$; luego pasados a MJ. ver Tabla 2 : columnas 1,2 y 3 . 


\begin{tabular}{|c|c|c|c|c|c|}
\hline & 1 & 2 & 3 & 4 & 5 \\
\hline & $\begin{array}{l}\text { Necesidades } \\
\text { energéticas } \\
\text { Diurna } \\
\mathrm{MJ} / \text { mes }\end{array}$ & $\begin{array}{l}\text { Necesidades } \\
\text { energéticas } \\
\text { Nocturna } \\
\text { MJ/mes }\end{array}$ & $\begin{array}{l}\text { Necesidades } \\
\text { energéticas } \\
\text { Total } \\
\text { MJ/mes }\end{array}$ & $\begin{array}{l}\text { Energía } \\
\text { disponible al } \\
\text { mes } \\
\mathrm{MJ} / \mathrm{m} 2\end{array}$ & $\begin{array}{l}\text { Energia solar } \\
\text { total } \\
\mathrm{MJ} / \mathrm{mes}\end{array}$ \\
\hline Enero & 3610,47 & 6017,44 & 9627,91 & 382,90 & 6509,32 \\
\hline Febrero & 3177,21 & 5295,35 & 8472,56 & 323,23 & 5494,90 \\
\hline Marzo & 6593,02 & 10988,37 & 17581,40 & 353,81 & 6014,70 \\
\hline Abril & 6630,70 & 11051,16 & 17681,86 & 260,76 & 4432,88 \\
\hline Mayo & 6906,98 & 11511,63 & 18418,60 & 248,07 & 4217,26 \\
\hline Junio & 7233,49 & 12055,81 & 19289,30 & 184,40 & 3134,80 \\
\hline Julio & 7534,88 & 12558,14 & 20093,02 & 178,51 & 3034,61 \\
\hline Agosto & 7534,88 & 12558,14 & 20093,02 & 246,55 & 4191,38 \\
\hline Septiembre & 6630.70 & 11051,16 & 17681,86 & 284.01 & 4828,22 \\
\hline Octubre & 6906,98 & 11511.63 & 18418.60 & 324,58 & 5517,88 \\
\hline Noviembre & 6329,30 & 10548,84 & 16878,14 & 332,25 & 5648,31 \\
\hline Diciembre & 3466,05 & 5776,74 & 9242,79 & 348,53 & 5924,95 \\
\hline $\begin{array}{l}\text { TOTAL } \\
\text { ANUAL }\end{array}$ & 72554,65 & 120924,42 & 193479,07 & 3467,60 & 58949,26 \\
\hline
\end{tabular}

Tabla 2. Valores de necesidades energéticas, energia disponible, energia solar

\section{SISTEMA DE CAPTACIÓN SOLAR} el año

Calculo de Energía Solar promedio en

A partir de la radiación solar horizontal incidente, Tabla 1, se aplica a este valor el Factor de corrección mensual por inclinación del panel (4), que para nuestro caso es 10 grados más que la latitud, esto es $45^{\circ}$ y con orientación hacia el NORTE, obteniéndose un valor corregido de la energía incidente en $\mathrm{kWh} / \mathrm{m} 2$ día, luego se pasan a $\mathrm{MJ} / \mathrm{m} 2$ día, se multiplica por el rendimien- to del colector, obtenido por datos del $\mathrm{fa}^{-}$ bricante y ajustados por temperatura ambiente, temperatura del agua e irradiancia solar dando un promedio del $80 \%$ y por los días del mes se obtiene la energía disponible al mes por $\mathrm{m} 2$, valores de la Tabla 2 columna 4.

Calculo de la cantidad de colectores necesarios

Para el cálculo de la cantidad de colectores necesarios, se buscó entre los proveedores locales varias alternativas y se decidió por el tipo de colector Marca Apricus 
de 30 tubos de tecnología de Heat Pipe, con un área de apertura de $2,84 \mathrm{~m} 2$ (5). La cantidad de colectores solares se calcula dividiendo las necesidades energéticas Diurnas en $\mathrm{MJ} / \mathrm{mes}$ por la energía disponible al mes en $\mathrm{MJ} / \mathrm{m} 2$, obteniéndose los metros cuadrados de colectores necesarios: 20,92 $\mathrm{m} 2$, que, divididos por el área de apertura del panel elegido, se obtiene la necesidad de 7,37 equipos. Dado la estructura de montaje para esta primera etapa se colocan 6 equipos, por lo cual la energía disponible al mes $\mathrm{MJ} / \mathrm{m} 2$ será la que se muestra en la columna 5 de la tabla 2 , lo que hace a un total $16374 \mathrm{KWh}$ y corresponde una cobertura del $81,24 \%$ de lo necesario para el período diurno. Tomando en cuenta el total de la energía necesaria para los dos periodos, es decir consumo de todo un día, este porcentaje de cobertura seria del $30 \%$.

\section{ANALISIS DE REDUCCIÓN EN LA EMISIÓN DE DIÓXIDO DE CARBONO}

Para calcular este dato se utilizó el factor de emisión de la red eléctrica Argentina (6), que nos dice cuántas toneladas de $\mathrm{CO} 2$ estamos emitiendo en nuestro país por cada MWh que generamos; para el año 2016 fue de 0,535 tCO2/MWh. En nuestro proyecto los $16374 \mathrm{KWh}$ ahorrados, arroja un valor de reducción de 8,762tn/ $\mathrm{CO} 2$ por año.

\section{ANÁLISIS DE RETORNO DE LA INVERSIÓN}

Considerando un costo de la energía eléctrica de 1,33\$/Kwh (05/03/18) y un porcentaje de aumento anual del $20 \%$, equivale a un retorno de la inversión de 10 años.

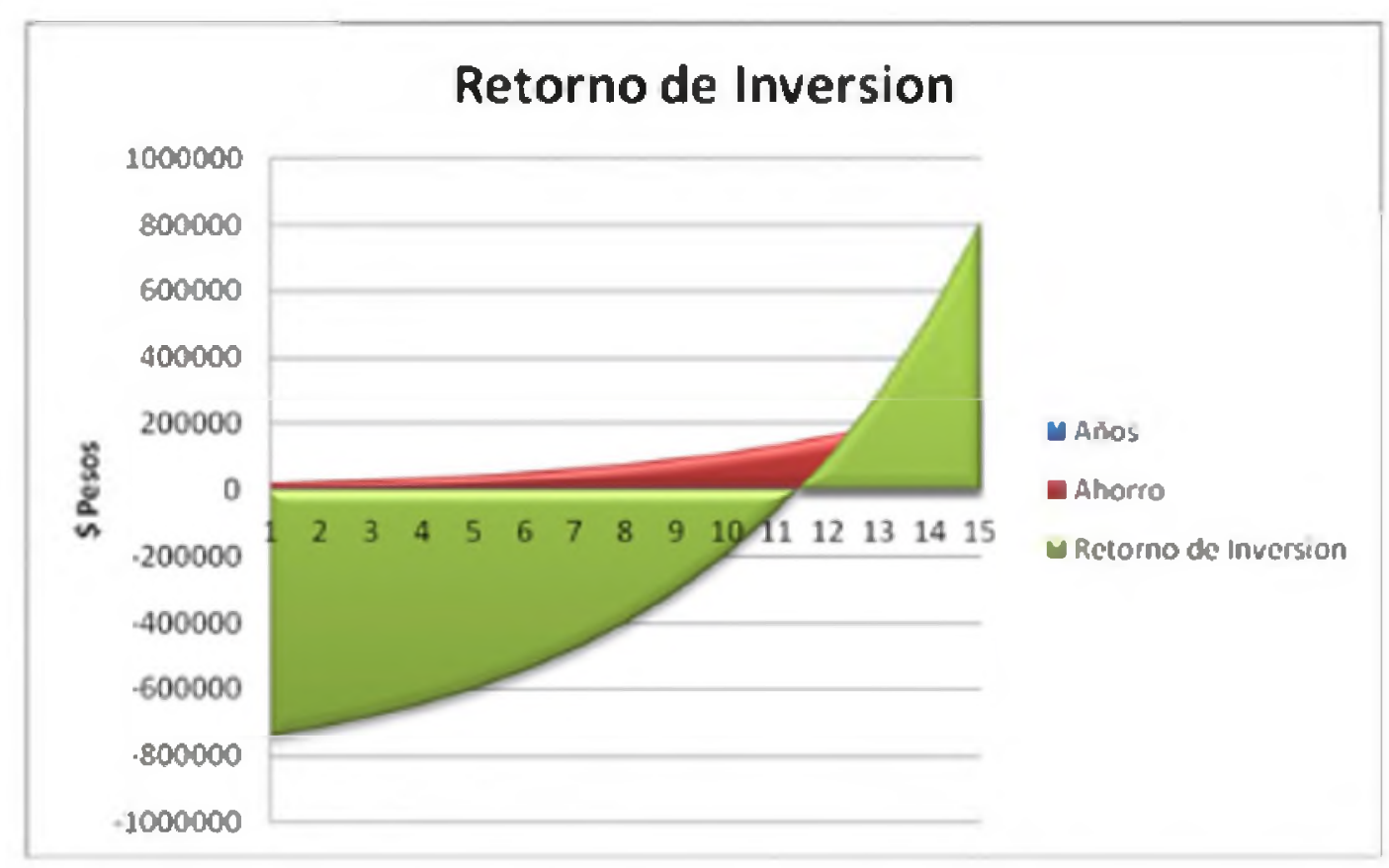

Figura 2 - Retorno de inversión 


\section{COMPARACIÓN CON LA SIMULACIÓN CON TRNSYS18}

Mediante el software de simulación Trnsys18 (7) se comprobaron los cálculos realizados anteriormente. En un principio se modelo el caso base, es decir, el calentamiento de agua a través de las resistencias eléctricas. Obteniendo como resultado la energía anual demandada por el sistema, ver esquema en la Figura 3.

Luego a dicho modelo se le agrego el sistema de colectores solares y se obtuvo así la energía anual aportada por los colectores solares, ver esquema en la Figura 4.

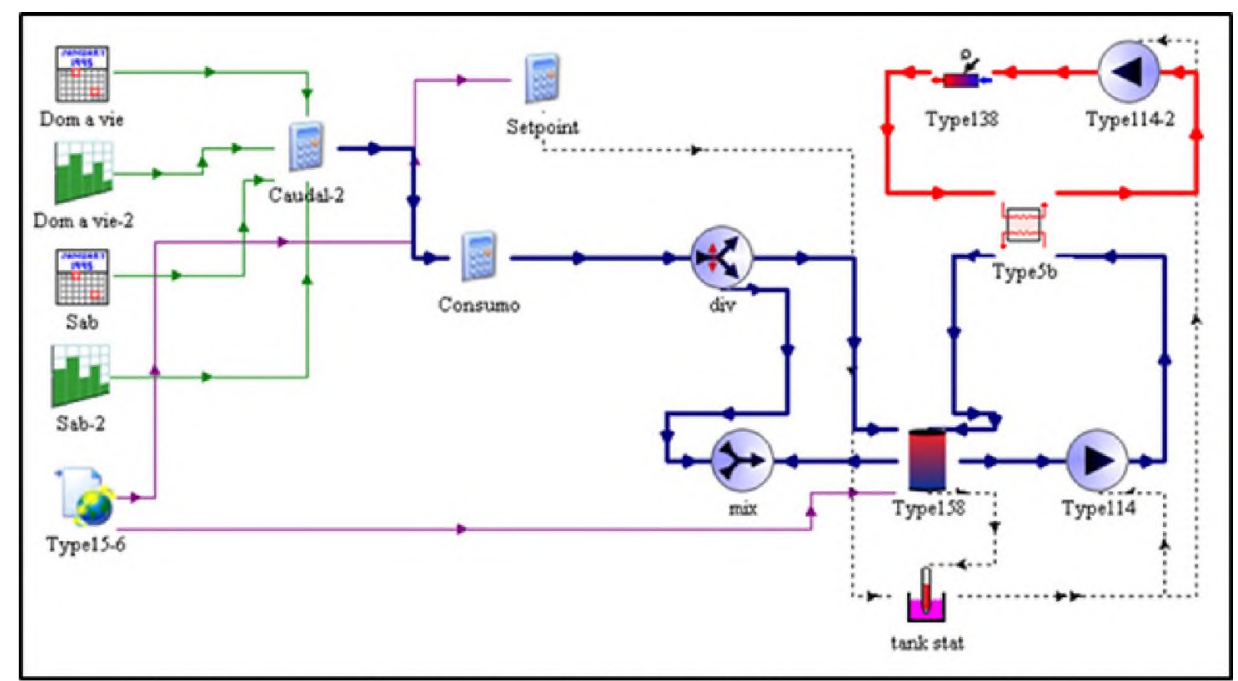

Figura 3 - Esquema de simulación en TRNSYS caso base, solo resistencias

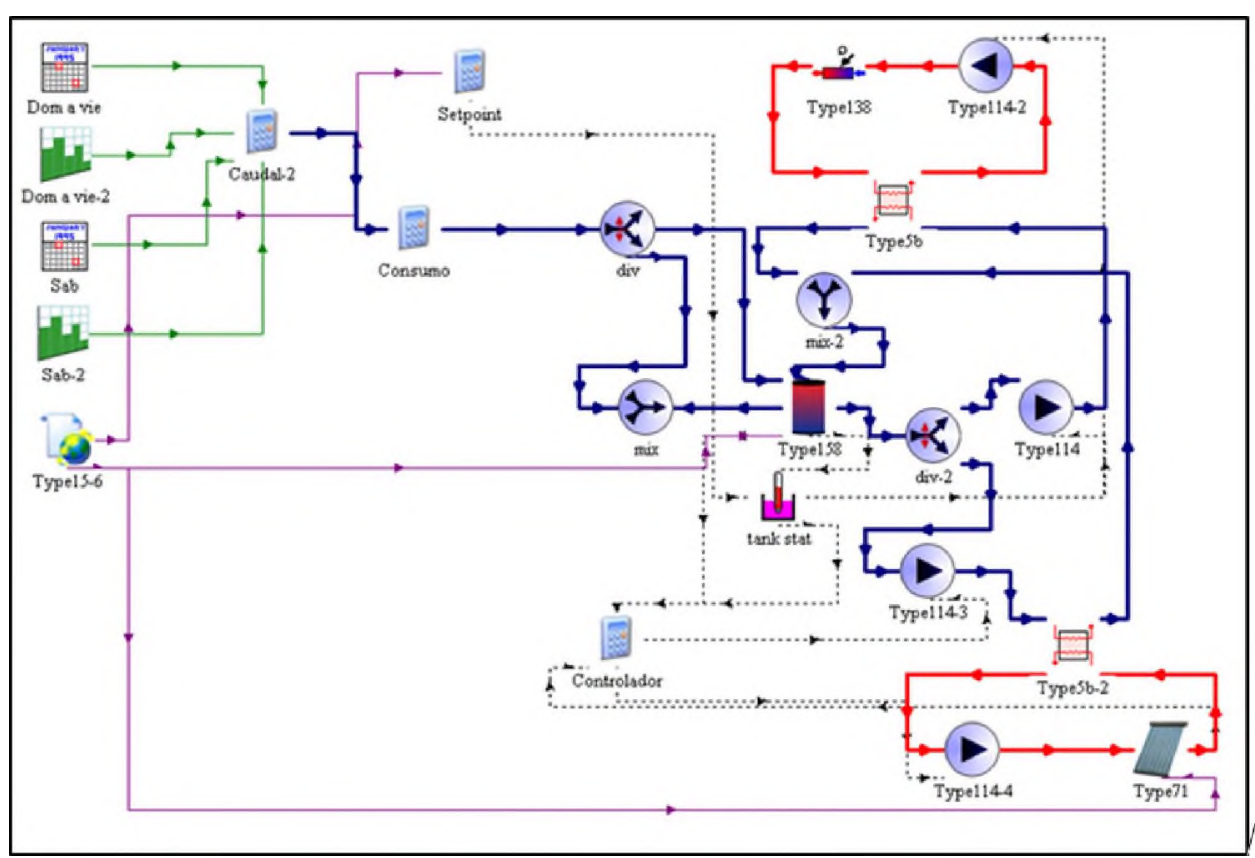

Figura 4 - Esquema de simulación en TRNSYS caso resistencias y paneles solares 
Como puede observarse en la Tabla posiblemente a los estados transitorios y 3, los valores de energía difieren de los variables climáticas que el software tiene calculados en forma convencional debido en cuenta para realizar los cálculos.

\begin{tabular}{|l|l|l|}
\hline Energía anual demandada (MJ) & 204453 & MJ \\
\hline Energía anual aportada por los colectores (MJ) & 49983 & MJ \\
\hline Porcentaje de sustitución & 23 & $\%$ \\
\hline
\end{tabular}

Tabla 3 -Datos simulados por el TRNSYS 18

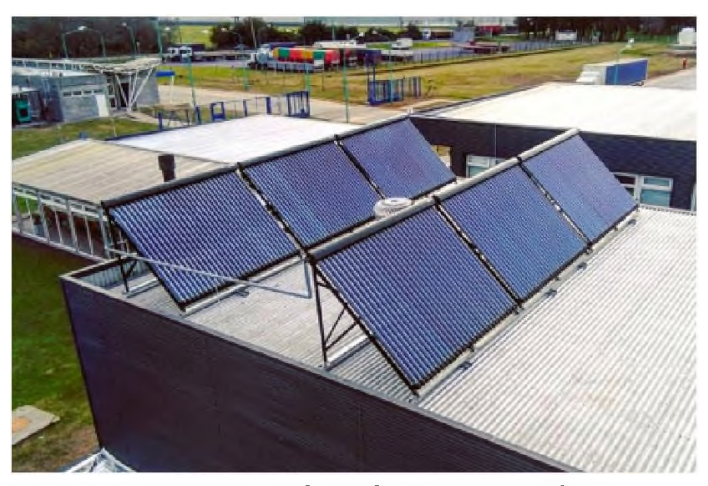

Figura 3 a) Instalación terminada

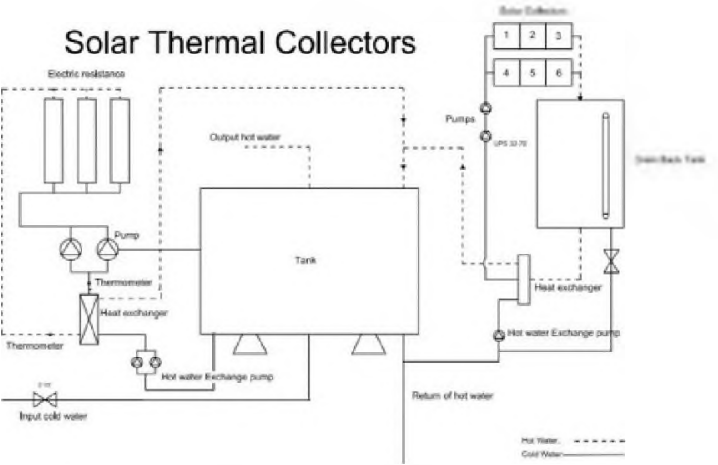

b) Esquema del sistema definitivo resistencias-colector solar

\section{CONCLUSIÓN}

Se logró un ahorro anual en energía eléctrica de $16374 \mathrm{kWh}$, que corresponde al $30 \%$ del total consumido, con un período de amortización de 10 años y dejar de emitir un total de $8,762 \mathrm{tn} / \mathrm{CO} 2$ por año. Además, este proyecto es un punto de partida para la empresa, en cuanto a la utilización y concientización sobre el uso de Energías Limpias y un incentivo para la realización de futuros proyectos con Energías Renovables.

\section{BIBLIOGRAFÍA}

(1) Norma UNE 94002:2005
(2) ATLAS DE ENERGÍA SOLAR DE LA REPÚBLICA ARGENTINA H. Grossi Gallegos Raúl Righini, Primera edición: mayo de 2007, ISBN: 978-987-9285-36-7

(3) Fuente: NASA ASDC

(4) Instalaciones de energía solar. Tomo II Tabla 6 pag.: 24, CENSOLAR España.

(5) Solar Water Heater - Solar Hot Water Solutions by Apricus. www.apricus.com

(6) Cálculo del Factor de Emisión de $\mathrm{CO} 2$ de la Red Argentina de Energía Eléctrica https://datos.minem.gob.ar/dataset/ calculo-del-factor-de-emision-de-co2-dela-red-argentina-de-energia-electrica.

(7) http://www.trnsys.com 